\title{
ON THE EDGE-HYPER-HAMILTONIAN LACEABILITY OF BALANCED HYPERCUBES
}

\author{
Jianxiang CaO, Minyong Shi \\ School of Computer Science \\ Communication University of China, Beijing, China \\ e-mail: jxcao@126.com, myshi@cuc.edu.cn \\ AND \\ LIHUA FENG ${ }^{1}$ \\ School of Mathematics and Statistics \\ Central South University, Changsha, China \\ e-mail: fenglh@163.com
}

\begin{abstract}
The balanced hypercube $B H_{n}$, defined by Wu and Huang, is a variant of the hypercube network $Q_{n}$, and has been proved to have better properties than $Q_{n}$ with the same number of links and processors. For a bipartite graph $G=\left(V_{0} \cup V_{1}, E\right)$, we say $G$ is edge-hyper-Hamiltonian laceable if it is Hamiltonian laceable, and for any vertex $v \in V_{i}, i \in\{0,1\}$, any edge $e \in E(G-v)$, there is a Hamiltonian path containing $e$ in $G-v$ between any two vertices of $V_{1-i}$. In this paper, we prove that $B H_{n}$ is edge-hyperHamiltonian laceable.
\end{abstract}

Keywords: balanced hypercubes, hyper-Hamiltonian laceability, edgehyper-Hamiltonian laceability.

2010 Mathematics Subject Classification: 05C38, 94C15.

\section{REFERENCES}

[1] J.A. Bondy and and U.S.R. Murty, Graph Theory with Applications (Macmillan Press, London, 1976). doi:10.1007/978-1-349-03521-2

\footnotetext{
${ }^{1}$ Corresponding author.
} 
[2] R.X. Hao, R. Zhang, Y.Q. Feng and J.X. Zhou, Hamiltonian cycle embedding for fault tolerance in balanced hypercubes, Appl. Math. Comput. 244 (2014) 447-456. doi:10.1016/j.amc.2014.07.015

[3] S.Y. Hsieh, G.H. Chen and C.W. Ho, Hamiltonian-laceability of star graphs, Networks 36 (2000) 225-232. doi:10.1002/1097-0037(200012)36:4〈225::AID-NET3〉3.0.CO;2-G

[4] K. Huang and J. Wu, Area efficient layout of balanced hypercubes, International Journal of High Speed Electronics and System 6 (1995) 631-646. doi:10.1142/S0129156495000237

[5] K. Huang and J. Wu, Balanced hypercubes, in: Proceedings of the 1992 International Conference on Parallel Processing 1 (CRC Press, 1992) 153-159.

[6] M. Lewinter and W. Widulski, Hyper-Hamilton laceable and caterpillar-spannable product graphs, Comput. Math. Appl. 34 (1997) 99-104. doi:10.1016/S0898-1221(97)00223-X

[7] H.Z. Lv and H.P. Zhang, Hyper-Hamiltonian laceability of balanced hypercubes, Journal of Supercomputing 68 (2014) 302-314. doi:10.1007/s11227-013-1040-6

[8] H.Z. Lv, X. Li and H.P. Zhang, Matching preclusion for balanced hypercube, Theoret. Comput. Sci. 465 (2012) 10-20. doi:10.1016/j.tcs.2012.09.020

[9] G.J. Simmons, Almost all n-dimensional rectangular lattices are Hamiltonian laceable, in: Proceedings of the 9th Southeastern Conf. on Combinatorics Graph Theory and Computing (Boca Raton, Fla., 1978) 103-108.

[10] D.B. West, Introduction to Graph Theory (Prentice Hall, 2001).

[11] J. Wu and K. Huang, The Balanced Hypercube: A cube-based system for fault-tolerant applications, IEEE Trans. Comput. 46 (1997) 484-490. doi:10.1109/12.588063

[12] J. Xu, Topological Structure and Analysis of Interconnection Networks (Kluwer Academic Publishers, Dordrecht, 2001). doi:10.1007/978-1-4757-3387-7

[13] M. Xu, X.D. Hu and J.M. Xu, Edge-pancyclicity and Hamiltonian laceability of balanced hypercubes, Appl. Math. Comput. 189 (2007) 1393-1401. doi:10.1016/j.amc.2006.12.036

[14] M.C. Yang, Bipanconnectivity of balanced hypercubes, Comput. Math. Appl. 60 (2010) 1859-1867. doi:10.1016/j.camwa.2010.07.016

[15] J.X. Zhou, Z.L. Wu, S.C. Yang and K.W. Yuan, Symmetric property and reliability of balanced hypercube, IEEE Trans. Comput. 64 (2015) 876-881. doi:10.1109/TC.2014.2304391

Received 24 June 2015

Revised 5 December 2015

Accepted 5 December 2015 